\title{
Human Nucleotide Excision Repair Syndromes: Molecular Clues to Unexpected Intricacies
}

\author{
J.H.J. Hoeijmakers
}

\section{INTRODUCTION}

THE DEVELOPMENT and maintenance of life has critically depended on the evolution of mechanisms that ensure genetic integrity and stability. DNA, the vital carrier of genetic information, is continually subjected to undesired chemical alterations. Numerous environmental or endogenous compounds and various types of radiation, such as X-rays and ultra-violet (UV) light, induce a wide variety of lesions in the bases, sugars or phosphates that make up the DNA. Obviously, such lesions (adducts, crosslinks, breaks, etc.) interfere with the proper functioning of the genome. An intricate network of DNA repair systems constitutes the main barrier protecting against the deleterious consequences of DNA injury. This is illustrated by the phenotype of inherited defects in one of these repair pathways. Such disorders are invariably associated with a characteristic hypersensitivity to a specific class of genotoxic agent. In addition, the DNA lesions that persist lead to cellular malfunction and to enhanced mutagenesis, due to the increased probability that errors occur upon replication of a damaged template. Somatic mutagenesis is the initiator of multistep carcinogenesis. Rare inborn disorders with hallmarks characteristic of repair defects comprise the class of chromosomal instability syndromes. Well-known examples are Fanconi's anaemia, ataxia telangiectasia and Bloom's syndrome, all of which display different manifestations of cancer predisposition and increased sensitivity to specific mutagens. The prototype disorder of this type is, however, xeroderma pigmentosum (XP), in which a defect in the nucleotide excision repair pathway underlies the pronounced predisposition to skin cancer and characteristic photosensitivity. The molecular mechanism and biological relevance of this repair system constitutes the topic of this chapter. For a comprehensive review of DNA damage and the intricate network of DNA repair systems in general, the interested reader is referred to Friedberg [1].

Nucleotide excision repair (henceforth abbreviated as NER) is one of the most versatile cellular repair mechanisms. This universal system eliminates a remarkably diverse array of structurally unrelated lesions, that range from UV-induced photoproducts (cyclobutane pyrimidine dimers and $6-4$ photoproducts) to bulky and small chemical adducts as well as intra-strand crosslinks. In this light, it is not surprising that the NER process entails multiple steps, and involves the concerted action of a number of proteins. The details of the reaction mechanism are best understood in the case of the UvrABC system in the

Correspondence to J.H.J. Hoeijmakers at the Dept. of Cell Biology and Genetics, Medical Genetics Centre, Erasmus University, P.O. Box 1738, 3000DR Rotterdam, The Netherlands. bacterium Escherichia coli (see [2-5] for recent reviews). Briefly, at least six distinct steps can be discerned: lesion recognition and lesion demarcation, which involve conformational changes in DNA, are carried out by the UvrA2B complex; a complex of UvrB and UvrC incises the damaged strand on both sides of the lesion at some distance, leaving the non-damaged strand intact; the damage-containing oligomer is removed by the helicase action of UvrD; and followed by gap-filling DNA synthesis by DNA polymerase I; the process is completed by the sealing of the new DNA to the pre-existing strand by DNA ligase. In principle, this mode of repair is error-free as it utilises the nucleotide sequence information of the intact complementary DNA strand.

\section{MOLECULAR INTRICACIES}

Although the outline and concept seem quite simple, it is becoming increasingly apparent that the scheme of the NER reaction mechanism, as outlined above for $E$. coli, represents a dramatic oversimplification when extrapolated to eukaryotes. This notion is based on a number of observations.

First, a minimum of two, in part, overlapping NER subpathways have been discovered. One subpathway, here referred to as transcription-coupled repair, deals with the complication that the vital process of transcription is blocked by lesions in the template. To cope with this urgent problem, transcriptioncoupled repair controls the preferential and complete elimination of injury in the transcribed strand of active structural genes (reviewed in [6, 7]). In this specialised NER subpathway, initial detection of damage occurs when RNA polymerase II becomes blocked in front of a lesion. As part of the reaction mechanism, the stalled RNA polymerase complex has to be displaced to give the repair machinery access to the injury. Another branch of the NER system, here designated global genome repair, accomplishes the removal of lesions in the remainder of the genome, including the non-transcribed strand of active genes. Damage recognition in this repair system is performed by a specific NER protein (complexes), and is for many (but not all) lesions slower and less efficient compared with transcriptioncoupled repair. The efficiency of damage recognition by the global genome repair system varies greatly from lesion to lesion, and may also vary with the chromatin conformation, the location in the genome and the state of cellular differentiation. This is not so surprising considering the tremendous task that is faced by this system in continually surveying the $2 \mathrm{~m}$ of DNA double helix in every mammalian nucleus for trace amounts of a diversity of lesions.

Secondly, as described below, genetic analysis of NER mutants and biochemical studies have provided evidence for the involvement of more than 20 gene products in the reaction 
Table 1. Main clinical features of NER syndromes

\begin{tabular}{lccc}
\hline & \multicolumn{3}{c}{ NER syndrome } \\
Symptoms and signs & XP* & CS $^{*}$ & PIBIDS \\
\hline Sun sensitivity & $+/++$ & + & + \\
Pigmentation abnormalities & + & - & - \\
Ichthyosis & - & - & + \\
Brittle hair/nails & - & - & + \\
Skin cancer & +++ & - & - \\
Caries & - & + & + \\
Accelerated neurodegeneration & $-l+\ddagger$ & $?$ & $?$ \\
Neurodysmyelination & - & + & + \\
Dwarfism & $-l \pm$ & + & + \\
Impaired sexual development & - & + & + \\
\hline
\end{tabular}

* Does not include patients with combined XP/CS. $\dagger>2000$-fold increase. $¥$ Patients belonging to XP group $C$ have no neurological abnormalities. XP, xeroderma pigmentosum; CS, Cockayne syndrome; PIBIDS, photosensitive trichothiodystrophy.

mechanism. These proteins have been conserved to a remarkable degree throughout eukaryotic evolution, underlining the fundamental importance of this process. This makes it likely that the mode of action of NER in lower eukaryotes, such as the baker's yeast Saccharomyces cererisiae, is probably to a large extent similar to that in man, although clear differences with the process in the prokaryotic model $(E$. coli $)$ are apparent.

Furthermore, intimate links between NER and other cellular processes have been disclosed, some of which were quite unexpected. Tight coordination of repair and cell cycle regulation exists: upon encountering abnormally high levels of damage, a transient block in cell cycle progression is introduced before DNA replication or prior to cell division. This gives the repair machinery the opportunity to remove the lesions before they give rise to permanent, potentially catastrophic changes in the genetic material. In addition, connections with recombination, replication, chromatin structure and the basic transcription apparatus have been unveiled.
In recent years, there has been spectactular progress in the identification of the components and protein complexes involved in eukaryotic NER, and details of the underlying molecular mechanism are emerging. Before discussing these, however, we will first turn to the human syndromes associated with defects in NER, which also reveal unexpected intricacies.

\section{CLINICAL INTRICACIES}

In addition to XP mentioned above, two other human disorders have been recognised as NER syndromes because of their diagnostic sun (UV) sensitivity: Cockayne syndrome and the photosensitive form of trichothiodystrophy, also designated PIBIDS. Remarkably, the common clinical denominator is limited to photosensitivity. In addition, a wide variety of distinct symptoms is associated with each of them. As discussed below, many of these features are difficult to rationalise with a defect in a DNA repair process. The main clinical, biochemical and genetic properties of these disorders are summarised in Tables 1 and 2.

XP patients display pronounced cutaneous manifestations. Besides acute photosensitivity, one observes strong pigmentation abnormalities, atrophy and more than a 2000-fold increment in all forms of skin cancer, including basal and squamous cell carcinomas and melanomas. These are strikingly limited to the sun-exposed parts, dramatically demonstrating the causative link with the genotoxic effects of the UV component of solar light. The frequency of cancer is also elevated for some internal tissues, but to a much lesser extent. A significant fraction of XP cases exhibit accelerated neurodegeneration, consistent with the concept that neurons are very dependent on proficient excision repair (Table 1). Cell hybridisation studies have led to the identification of seven excision-deficient complementation groups within the population of XP patients (designated XP-A to XP-G; Table 2) [8]. This implies that at least seven distinct genes are involved in this disorder. Additionally, a separate category of XP individuals, with a presumed defect in the poorly defined postreplication repair process, but with apparently normal NER is known. This group

Table 2. Properties of XP,CS and PIBIDS complementation groups

\begin{tabular}{|c|c|c|c|c|c|c|c|}
\hline \multirow{2}{*}{$\begin{array}{l}\text { Complementation } \\
\text { group }\end{array}$} & \multirow{2}{*}{${ }_{\text {sensitivity }}^{\text {UV }}$} & \multirow{2}{*}{$\begin{array}{l}\text { Residual } \\
\text { UDSt }\end{array}$} & \multicolumn{2}{|c|}{ NER defect* } & \multirow[b]{2}{*}{ Relative frequency } & \multirow{2}{*}{$\begin{array}{c}\text { Overlap with } \\
\text { other NER } \\
\text { syndrome }\end{array}$} & \multirow[b]{2}{*}{ Overlap with rodent group } \\
\hline & & & TCR & GGR & & & \\
\hline XP-A & ++ & $<5 \%$ & + & + & High & No & Not groups 1-7, 11 \\
\hline XP-B & ++ & $<10 \%$ & + & + & Three families & CS, PIBIDS & Rodent group 3 \\
\hline $\mathrm{XP}-\mathrm{C}$ & + & $15-30 \%$ & - & + & High & No & Not groups 1-6 \\
\hline XP-D & $+t$ & $15-50 \%$ & + & + & Intermediate & CS, PIBIDS & Rodent group 2 \\
\hline XP-E & \pm & $>50 \%$ & $?$ & $?$ & Rare & No & Not groups $1-3,5$ \\
\hline XP-F & + & $15-30 \%$ & + & + & Rare & No & Rodent group 4? \\
\hline XP-G & $+t$ & $<10 \%$ & + & + & Rare & CS & Rodent group 5 \\
\hline TTD-A & + & $15 \%$ & $+(?)$ & + & One family & & Not groups $1-3,5,6$ \\
\hline CS-A & + & wt & + & - & Rare & No & Not known \\
\hline CS-B & + & wt & + & - & High & No & Rodent group 6 \\
\hline XP-V¥ & + & wt & - & - & High & No & No NER defect \\
\hline
\end{tabular}

* TCR, transcription-coupled repair; GGR, global genome repair; +, NER subpathway is defective; -, NER subpathway functional. † UDS, unscheduled DNA synthesis (expressed as percentage of UDS of normal cells). $\ddagger$ XPV, XP-variant, defective post replication repair, NER normal. wt, wildtype; TTD, trichothiodystrophy. 
is called the XP-variant (XP-V) and will not be discussed further here. For a comprehensive review on the clinical manifestations of XP see Cleaver and Kraemer [9].

The classical form of Cockayne syndrome (CS) is characterised by severe neurological dysfunction (which is, in contrast to XP, primarily related to dysmyelination), growth and developmental abnormalities, dental caries, wizened appearance and bird-like facies. The cutaneous symptoms are limited to sun sensitivity. A remarkable feature addressed below is that CS patients do not display clear cancer predisposition (Table 1). Complementation analysis has defined two CS groups within the classical form of the disease: CS-A and CS-B (Table 2) [10, 11]. The non-classical form of CS defines extremely rare individuals with simultaneous manifestations of XP and CS. Patients within this subcategory are assigned to XP groups B, D and G (Table 2) [8, 12]. These are also the NER complementation groups which exhibit the largest clinical heterogeneity. An extensive overview on the manifestations of CS can be found elsewhere [13].

PIBIDS is an acronym for a specific combination of symptoms: photosensitivity, ichthyosis, brittle hair and nails, impaired intelligence, decreased fertility and short stature. It is a special form of a much broader syndrome called Trichothiodystrophy (TTD) that also encompases IBIDS, BIDS and SIBIDS patients. The clinical hallmark of TTD is brittle hair, which is due to a reduced content of a class of matrix proteins with an ultra-rich cysteine contact that provides hair with its natural strength. The neurological dysfunction is related to neurodysmyelination, as in CS. Only the photosensitive subset of TTD patients displays a NER defect ([14] and references therein). Cell fusion and microinjection of cloned NER genes has also disclosed genetic heterogeneity within this NER syndrome. Intriguingly, there is again considerable overlap with XP, although the clinical features are very different. Most PIBIDS cases fall into the same group as XP-D. Recently, one PIBIDS family was found to belong to XP-B, and a third kindred constitutes a distinct NERdeficient complementation group, TTD-A, not (yet) associated with XP (Table 2) $[15,16]$. At the clinical level, it is now becoming increasingly evident that $\mathrm{CS}$ and PIBIDS resemble each other much more than previously anticipated (see [17] and references therein). This concerns the neurological, developmental (physical and sexual) problems, cutaneous abnormalities and the remarkable absence of cancer predisposition (Table 1 ). A complicating factor is that a large fraction of the TTD patients exhibits essentially all of the symptoms of PIBIDS, except for the photosensitivity, but has no overt repair defect.

Thus, both genetic heterogeneity within and genetic overlap between all NER disorders is found. A specific subset of XP groups (notably XP-D and XP-B) is associated with extreme clinical variability ranging from XP to $C S$ and PIBIDS. As argued at the end of this review, these disorders may in fact be considered different manifestations of one heterogeneous clinical continuum. In this context, we will also address the puzzling connection between defective excision repair and features such as brittle hair and nails, neurodysmyelination and impaired sexual development.

\section{OTHER EUKARYOTIC NER MUTANTS}

In addition to the naturally occurring human disorders, a large collection of rodent (mainly Chinese hamster) UV-sensitive cell mutants has been generated in the laboratory. Complementation analysis by cell fusion has identified 11 NERdeficient complementation groups among them [18] (see also Busch and associates [19] and references therein). Mutants of groups $1-5$ are the most defective in NER and consequently display the most pronounced UV-sensitivity. Mutants of groups 1,4 and to a lesser extent 11 also exhibit an extreme sensitivity to crosslinking agents. A subset of rodent mutants appeared to be a very valuable source for the isolation of human NER genes that correct their repair defect. These genes are termed ERCC genes (for excision repair cross-complementing followed by a number that refers to the corrected rodent complementation group). In this way, $E R C C 1-6$ have been cloned. Importantly, many of these genes were discovered to be implicated in human NER disorders (Table 2). Thus, the ERCC2 and 3 genes were found to be responsible for XP groups $D$ and $B$, respectively $[20,21]$. ERCC5 corrects the UV sensitivity of XP-G [22, 23], whereas the ERCC6 gene was found to be mutated in CS-B [24]. Similar experiments on ERCCI failed to provide a link with any of the known XP, CS and TTD complementation groups [25] (and Vermeulen, unpublished results). Hence, this gene does not (yet) seem to be represented among the identified human NER disorders. A similar analysis for the recently cloned ERCC4 gene (Thompson, personal communication) is in progress, but other findings make XP-F a likely candidate [26, 27].

Finally, another relevant class of eukaryotic NER mutants is presented by the rad 3 epistasis group of $S$. cerevisiae. Within this category of UV-sensitive yeast mutants, at least 15 complementation groups have been identified. The versatility of yeast genetics has permitted the cloning of almost all of the corresponding genes, many of which were found to have a mammalian counterpart (Table 3 ). The strong parallels with the mammalian system that have emerged in recent years make this organism a relevant paradigm for human NER (see [5, 27a, 28, 29] for reviews on yeast NER).

\section{THE BIOCHEMICAL DEFECT IN MAMMALIAN NER MUTANTS}

As in yeast and $E$. coli, a large proportion of the human and rodent mutants display defects in both the transcription-coupled and global genome subpathways, indicating that their mutation affects a step common to or necessary for both modes of NER (see also Table 2). This includes mutants of rodent groups 1-5, and probably 11, and XP groups A, B, D, F, G and TTD-A. Biochemical analysis has revealed that incision is completely or strongly impaired. This suggests, but does not prove, that the mutated proteins are involved in early pre-incision stages of the NER reaction. Mutants with disturbances in only one of the NER subpathways exhibit an intermediate phenotype, in terms of sensitivity and removal of lesions from different parts of the genome (Table 2). XP group C carries a defect limited to the global genome repair [30]. CS groups A and B as well as rodent group 6 are selectively affected in transcription-coupled repair $[31,32]$. The fact that XP-C is associated with strong cancer predisposition suggests that the global genome subpathway is of major importance for preventing mutagenesis and carcinogenesis. This may provide a plausible explanation why, in CS, no significant cancer predisposition is observed; CS patients still possess a potent global genome repair system. Furthermore, the average age reached by CS patients is only 12.5 years, which is quite young in terms of oncogenesis. The transcription-coupled repair subpathway may be important for cell survival, but less relevant for preventing mutagenesis.

\section{MAMMALIAN NER GENES AND THEIR FUNCTION: A POSSIBLE MODEL FOR THE NER REACTION}

A large fraction of the important NER genes involved in the seven XP complementation groups has been molecularly cloned. 
Table 3. Main properties of cloned human NER genes

\begin{tabular}{|c|c|c|c|c|c|}
\hline Gene* $^{*}$ & $\begin{array}{l}\text { Chromosomal } \\
\text { location }\end{array}$ & $\begin{array}{l}\text { Gene }(k b) \\
\text { size }\end{array}$ & $\begin{array}{l}\text { Protein } \\
\text { size }(a a) \dagger\end{array}$ & $\begin{array}{c}\text { Yeast } \\
\text { homologue }\end{array}$ & Protein properties $\ddagger$ \\
\hline$X P A$ & $9 q 34$ & $\sim 25$ & 273 & rad14 & $\begin{array}{l}\mathrm{Zn}^{2+} \text {-finger, binds damaged DNA, transient interaction with ERCC1 } \\
\text { complex }\end{array}$ \\
\hline$X P B / E R C C 3$ & $2 \mathrm{q} 21$ & $\sim 45$ & 782 & $\operatorname{rad} 25(s s l 2)$ & $\begin{array}{l}3^{\prime} \rightarrow 5^{\prime} \text { DNA helicase, essential for transcription initiation, part of BTF } 2 / \\
\text { TFIIH }\end{array}$ \\
\hline$X P C$ & $3 \mathrm{p} 25.15$ & $?$ & 940 & $\operatorname{rad4} ?$ & $\begin{array}{l}\text { Limited homology to RAD4, complexed with HHR23B, strong ss-DNA } \\
\text { binding, involved in GGR only }\end{array}$ \\
\hline$X P D / E R C C 2$ & $19 \mathrm{q} 13.2 \$$ & $\sim 20$ & 760 & $\operatorname{rad} 3$ & $\begin{array}{l}5^{\prime} \rightarrow 3^{\prime} \text { DNA helicase, essential for transcription initiation, part of BTF } 2 / \\
\text { TFIIH }\end{array}$ \\
\hline$X P E$ & 11 & $?$ & 1140 & Not known & $\begin{array}{l}\text { Binds UV-damaged DNA, no causative mutations identified in XP-E } \\
\text { patients yet }\end{array}$ \\
\hline$X P G / E R C C 5$ & $13 q 32-33$ & $\sim 32$ & 1186 & $\operatorname{rad} 2$ & Branched DNA-specific ss-endonuclease, 3 '-incision \\
\hline$C S B / E R C C 6$ & $10 q 11-21$ & $\sim 85$ & 1493 & $\operatorname{rad26}$ & $\begin{array}{l}\text { DNA helicase for displacement of stalled RNA polymerase? nonessential } \\
\text { function, involved in TCR only }\end{array}$ \\
\hline ERCCI & $19 \mathrm{q} 13.2 \$$ & $15-17$ & 297 & rad10 & $\begin{array}{l}\text { C-terminal homology to parts of UvrA and C, involved in recombination? } \\
\text { In complex with } E R C C 4,11, \mathrm{XPF} \text {, ss-endonuclease as complex? } 5 \text { '- } \\
\text { incision? }\end{array}$ \\
\hline ERCC4 & $16 \mathrm{p} 13.3$ & $?$ & $?$ & radl & $\begin{array}{l}\text { May be identical to XPF, in complex with } E R C C 1 \text { and } 11 \text {, ss-endonuclease } \\
\text { as complex? }\end{array}$ \\
\hline$H H R 23 A$ & $19 \mathrm{pl} 13.2$ & $?$ & 363 & $\operatorname{rad} 23$ & Ubiquitin-like $\mathbf{N}$-terminus. \\
\hline$H H R 23 B$ & $3 \mathrm{p} 25.1 \S$ & $?$ & 409 & $\operatorname{rad} 23$ & $\begin{array}{l}\text { Ubiquitin-like N-terminus, complexed with XPC, as complex strong } \\
\text { ssDNA-binding and involved in GGR only }\end{array}$ \\
\hline$p 62^{B T F 2}$ & $11 \mathrm{p} 14-15.1$ & $?$ & 548 & $t f h l$ & Part of BTF2/TFIIH, essential for transcription initiation \\
\hline$p 44^{B T F 2}$ & $5 q 1.3$ & $?$ & 395 & ssll & $\begin{array}{l}\text { DNA-binding } \mathrm{Zn}^{2+} \text {-finger? Part of BTF2/TFIIH, essential for } \\
\text { transcription initiation }\end{array}$ \\
\hline$p 34^{B T F 2}$ & $?$ & $?$ & 303 & Not known & $\mathrm{Zn} 2+$-finger? Part of BTF2/TFIIH, essential for transcription initiation \\
\hline
\end{tabular}

*Not included in this table are the genes for proteins involved in the DNA-synthesis step of the NER reaction, such as PCNA, hSSB, DNA ligase, etc. $\nmid$ aa, amino acids. $\ddagger$ Question marks indicate properties inferred but not proven. TCR, transcription-coupled repair; GGR, global genome repair. $\int$ The $X P C$ and $H H R Z 3 B$ genes may share a common $650-\mathrm{kb}$ MluI fragment; $E R C C I$ and $X P D / E R C C 2$ are less than $250 \mathrm{~kb}$ apart.

In addition, one of the two genes implicated in the classical form of CS has been isolated, as have several NER genes for which no human NER complementation groups are yet known, but for which either a rodent mutant or a yeast NER equivalent exists. Furthermore, using an in vitro NER assay system based on cellfree extracts, several proteins known to be involved in DNA replication have also been demonstrated to participate in the DNA synthesis step of the NER reaction.

Computer-assisted comparison of the predicted amino acid sequence of the encoded proteins, with known gene products present in large databases, has highlighted a striking resemblance with NER proteins of the yeast rad3 epistasis group. The extent of similarity suggests a 'golden rule', namely that for each yeast NER protein there is a counterpart in man and vice versa. The conclusion must be that the entire NER reaction mechanism is strongly conserved in all eukaryotes. The primary amino acid sequence also disclosed homology with functional protein domains, such as well-characterised DNA-binding and helixunwinding sequence motifs. This type of information provided valuable clues to the biochernical activity of the encoded polypeptides. Finally, overproduction, purification and enzymological characterisation has led to the elucidation of functional properties of several of the NEK proteins and the identification of intricate complexes. Table 3 lists all mammalian NER genes cloned to date and summarises their main properties.

The current knowledge of NER proteins identified thus far can be tentatively integrated into a possible NER reaction mechanism. This still highly speculative model is depicted in EงC 30:13-8
Figure 1A-F, and each step is discussed below, in terms of the available evidence and our still considerable ignorance.

\section{Damage recognition (Figure $1 A, T$ able 3 )}

A prime candidate for the universal damage recognition factor of NER is the XPA protein, possibly with the assistance of the XPE factor.

The XPA gene codes for a 273 amino acid protein with the sequence hallmarks of a DNA-binding $\mathrm{Zn}^{2+}$-finger domain [33]. The gene product has been purified, and biochemical evidence has confirmed that it is a zinc metalloprotein with affinity for double-stranded (ds) and single-stranded (ss) DNA [34-37]. A marked preference has been found for a number of lesions, including 6-4 photoproducts and Cs-Pt adducts, with poor binding to cyclobutane pyrimidine dimers $[35,38]$. This parrallels strikingly the preference of the NER pathway itself, and is reminiscent of the lesion-binding spectrum of the $E$. coli $\mathrm{UvrA}_{2} \mathrm{~B}$ complex. Using the in vitro cell-free NER assay, the XPA product has been functionally assigned to a step in the preincision stage of the reaction [39]. These data are consistent with the idea that XPA is implicated in damage recognition.

The $127-\mathrm{kDa}$ protein associated with the XP-E defect was purified in different laboratories, based on its strong affinity for UV-damaged DNA substrates and its high abundance [40-43]. Microinjection of the protein in XP-E cells induced wildtype levels of UV-dependent repair synthesis [44]. The latter finding strongly suggests that it is responsible for XP-E. However, direct proof that mutations in the gene cause the XP-E defect is 


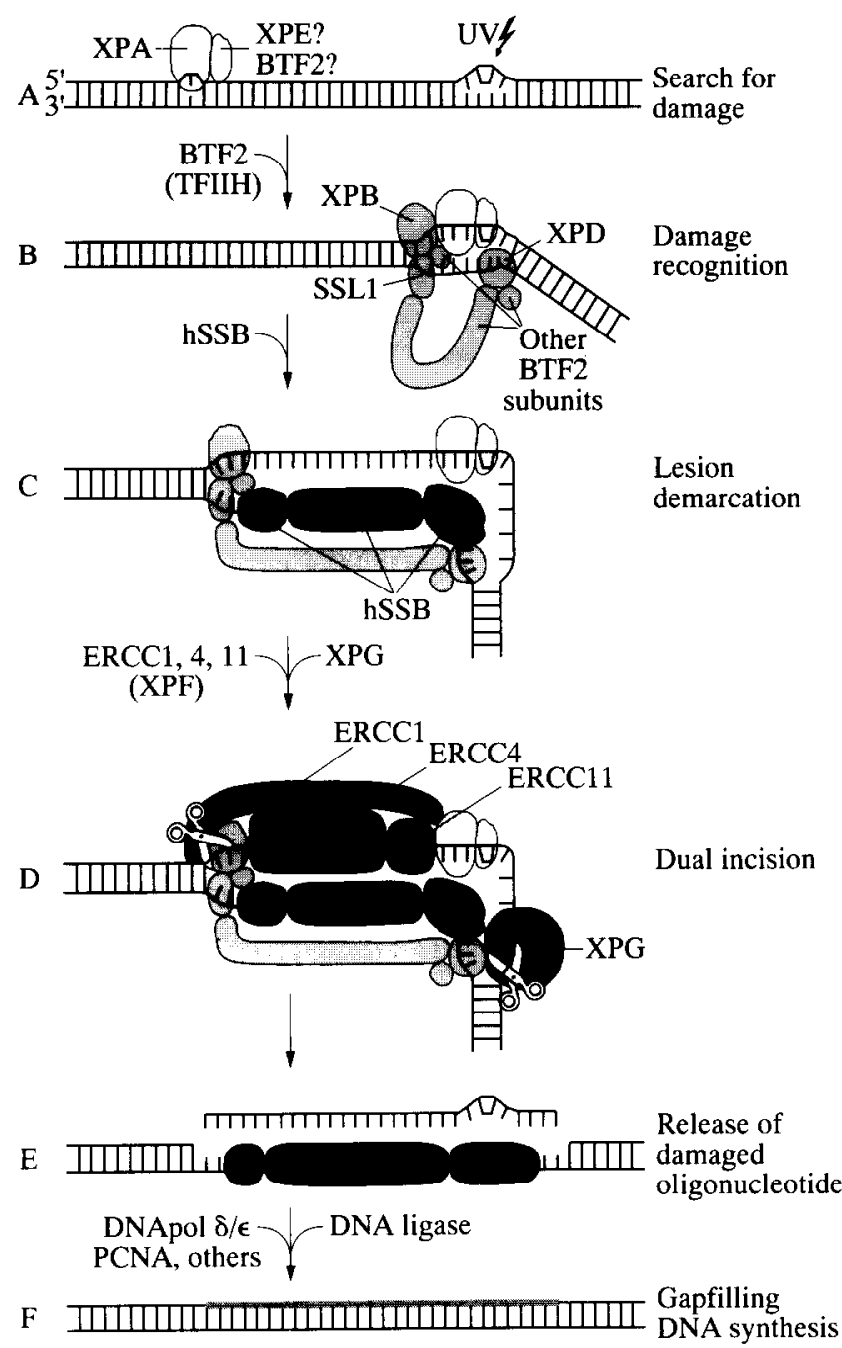

Figure 1. Hypothetical model for the molecular mechanism of the mammalian nucleotide excision repair reaction. Sequential steps are indicated with A-F. See text for a detailed explanation.

still lacking. The deduced amino acid sequence does not provide any clues to the function of the cncoded protein, nor has any yeast homologue been identified [43]. The XP-E correcting factor has a strong preference for binding to DNA containing 64 photoproducts, with modest discrimination of cyclobutane pyrimidine dimers and no measurable affinity for psoralenthymine monoadducts [45]. These lesion-binding characteristics are not consistent with it being the universal damage recognition factor, but an auxiliary role cannot be ruled out. The striking abundance of the protein in normal cells (in the order of $10^{5}$ copies per cell [42]), suggests that it has an additional function. A general characteristic of NER factors is that they seem to be present only in trace amounts. One possibility is that the XP-E correcting protein assists XPA in lesion detection, but direct interaction between these polypeptides has not been demonstrated. Alternatively, recent evidence suggests that the XPA protein may associate transiently with ERCCl $([46,47]$, De Laat $e t a l$., unpublished results, see below), although in crude cell extracts, these proteins are not significantly complexed to each other (Van Vuuren et al., unpublished results).

Detection of the primary lesion in the transcription-coupled NER subpathway is probably performed by RNA polymerase
II. The transcription machinery presumably subjects the DNA to a more rigorous test for intactness than achieved via the standard NER damage recognition complex. This explains why lesions that are poorly removed from the genome overall are efficiently eliminated by the transcription-coupled repair mode.

\section{Demarcation of the lesion (Figure $1 B-D$ )}

By analogy with the $E$. coli system, it is plausible that after damage detection the DNA and chromatin have to be made accessible for recognition by the incision protein complex(es). This may involve the induction of a strongly kinked, locally unwound, but for all lesions uniform, DNA structure as observed with the $\operatorname{UvrA}_{2} B$ complex, which overrides the aherrant lesion-specific conformation. Currently, no proteins have been shown to be associated with this presumed NER reaction intermediate. The hSSB complex, composed of three subunits of 70, 34 and $11 \mathrm{kDa}$, previously found to be involved in initiation and elongation of DNA replication, may act at this stage. From studies involving the cell-free in vitro NER assay, it appears that this trio of ss DNA-binding proteins plays a role prior to or at the incision step $[39,48]$. As indicated in Figure $1 \mathrm{C}$, this complex fits well in a model involving a denatured intermediate. An additional participant in this stage of the reaction could be the BTF2/TFIIH transcription/repair complex which possesses bidirectional helicase activity discussed below.

The role of the BTF2/TFIIH transcription-repair complex (Figure $1 B-D$, Table 3). Many parallels exist between XPD/ ERCC2 and XPB/ERCC3 (and their yeast counterparts rad 3 and $\operatorname{rad} 25$, also known as ssl2) [21]. Based on the identification of helicase motifs in the primary amino acid sequence, Weeda and colleagues proposed that both proteins form a complex with bidirectional helix-unwinding activity [21]. As apparent below, this early idea was corroborated by recent studies. The notion that null-alleles of one or both genes in yeast $[49,50]$, Drosophila [51] and mouse (Weeda et al., unpublished results) are nonviable indicates that these repair proteins must be involved in an additional process essential for viability. The nature of the latter was elucidated by a surprising discovery made by Egly and coworkers [54], who were studing transcription initiation, an intricate process like NER, involving the concerted action of numerous gene products (for a review see [53]). A multi-subunit component, requircd in a late stage of RNA polymerase II basal transcription initiation, is BTF2, also designated TFIIH. It is a complex of at least six polypeptides [54, 55]. The two largest subunits of this basal transcription factor were found to be the repair proteins XPB/ERCC3 [52] and XPD/ERCC2 [56, 57]. Moreover, purified BTF2 corrects the XP-B, XP-D and TTDA repair defects in vivo, as well as in vitro $[16,58]$, indicating the presence of at least three repair proteins in this transcription factor, and a striking relationship with NER complementation groups involving TTD. Furthermore, the p44 BTF2 subunit has recently been identified as the human homologue of the yeast ssll protein [59] (see also Table 3). Mutations in this yeast gene, originally thought to be involved in translation, give rise to a UV-sensitive phenotype [60] consistent with a function in repair. Finally, a dominant mutation in the $X P B / E R C C 3$ gene and antibodies against XPD protein completely abrogate NER and transcription in vivo $[16,58]$. This establishes the unique dual functionality of the complex in repair and basal transcription, and extends it to the in vivo situation.

Purified BTF2 displays the following activities and properties: a DNA-dependent ATPase, a protein kinase phosphorylating 
the carboxy-terminus of the large subunit of RNA polymerase II, and a bidirectional IDNA unwinding activity ([56] and references therein). The latter is due to the XPB/ERCC3 $\left(3^{\prime} \rightarrow 5^{\prime}\right)$ and the XPD/ERCC2 $\left(5^{\prime} \rightarrow 3^{\prime}\right)$ helicases. In addition, the BTF2 complex is endowed with two proteins (p44 and p34) containing one or more $\mathrm{Zn}^{2+}$-finger domains that may mediate DNA and/or protein-protein interactions [59] (Table 3). Studies using in vitro transcription initiation assays have not fully established the role of BTF2 in transcription initiation. However, several plausible models can be advanced. The bidirectional helicase activity may be required for inducing a specific DNA conformation by locially opening the template for loading RNA polymerase on to the transcribed strand. Alternatively, or in addition, the complex may promote promoter clearance [61].

What possible role could the BTF2 complex have in NER? Since BTF2 repair mutants show defects in both transcriptioncoupled as well as global genome repair (Table 2), the complex probably functions in the core of the NER reaction. Furthermore, it is reasonable to suppose that BTF2 catalyses a similar step in the context of NER and transcription initiation. Thus, one of the options is that it induces a denatured DNA conformation which is required for loading a NER incision complex or a lesion-detection complex on to the template and/or for altering the DNA conformation around the lesion in a manner similar to Uvr $A_{2} B$ (see Figure $1 A-C$ ). Alternatively or in addition, this complex may be involved in clearance of the damaged region, possibly including release of the damage-containing oligonucleotide and turnover of repair proteins (Figure 1D-E).

\section{Dual incision (Figure 1D, Table 3)}

Huang and coworkers elegantly demonstrated that the mammalian NER machinery makes a dual incision in the damaged strand, involving the fifth phosphodiester bond 3' and the 21-23 bond $5^{\prime}$ of the lesion [62]. Two strong candidates for this step have recently been proposed. The first is a protein complex consisting of the ERCC1, ERCC4, ERCC11 and XPF proteins (the latter may be identical to either ERCC4 or ERCC11) [26, 27] (see also Table 3). The yeast counterpart of this complex, comprising rad1 and 10 prcteins, has bccn shown to posscss a ss DNA endonuclease activity', that can act on unwound regions of negatively supercoiled DNA [63]. This complex is also implicated in intra-chromosomal mitotic recombination. Both processes encompass DNA incision as an obligatory step in their reaction mechanism. By analogy to yeast, the human ERCC1 complex is also predicted to exhibit ss DNA incision activity. A second endonuclease activity associated with a repair factor was reported for the rad2 [64] and human XPG/ERCC5 proteins (Wood, personal communication). In contrast to the radl-radl0 complex, the rad2 endonuclease acts only on ss DNA. Considering the fact that both proteins require a ss DNA substrate, it is feasible that the DNA around the lesion is locally unwound. The bidirectional helicase activity of the BTF2 complex is an excellent candidate for this function. Association of the hSSB protein complex may prevent the denatured region from reannealing, and thus facilitate the first incision by the rad2-XPG endonuclease. This is followed by the second cleavage generated by the radl-ERCC1 complex, completing the dual incision reaction. From recent studies identifying XPG-rad2 as an endonuclease specific for branched DNA structures, the XPG incision is most likely $3^{\prime}$ to the lesion [65].

\section{Post incision events (Figure $1 E-F$ )}

The final stages of the NER reaction should entail release of the damage-containing oligomer, turnover of the bound NER proteins, gap filling DNA synthesis and sealing of the remaining nick by ligation. Currently, it is unknown which protein(s) carries out the first of these steps. Again, the BTF2 complex is a potential candidate since it harbours two helicases that could peel off the injury-containing 27-29-mer. Alternatively, it is possible that the DNA replication machinery carries out this reaction.

The use of the cell-free NER assay has permitted the identification of the proliferating cell nuclear antigen (PCNA) as being required for the gap-filling DNA synthesis step [39, 66]. PCNA is known to stimulate DNA polymerase delta and/or epsilon in regular DNA replication. Thus, the implication of PCNA involvement in this part of the NER reaction mechanism suggests that the repair synthesis itself is mediated by any or both of these polymerases. The final sealing of the new DNA to the pre-existing strand is thought to be carried out by DNA ligase I.

Steps specific for the global genome repair subpathway (Table 3). Analysis of mutants has lead to the identification of at least three genes selectively implicated in the repair of the non-transcribed bulk of the genome: $\mathrm{rad} 7$ and rad16 in yeast [67] and XPC in $\operatorname{man}[30]$.

The XPC amino acid sequence displays significant homology to the yeast rad4 protein $[68,69]$. However, rad4 null mutants are, in contrast to XP-C mutants, defective in both NER subpathways [67]. This may reflect a principal difference between mammals and yeast. The recently purified XPC protein (125 kDa) appears to be complexed with HHR23B (43 kDa), one of the two human homologues of yeast rad23. Intriguingly, the $X P C$ and $H H R 23 B$ genes are also located very close to each other in the genome (at chromosome $3 \mathrm{p} 25$, probably within $650 \mathrm{~kb}$ ) [70]. The XPC-HHR23B heterodimer has a very high affinity for ssDNA, but no associated enzymatic activities have been detected [69]. A notable feature of the HHR23 proteins is the presence of a ubiquitin-like domain in the $\mathrm{N}$-terminus [69]. In some other ubiquitin-fusion proteins, this moiety is thought to function as a chaperone, facilitating complex formation. Hence this domain may perform a similar role in assembling the XPC-HHR23B complex.

In keeping with the golden rule delineated from the striking correspondence between yeast and mammalian NER, it would be expected that human homologues of $\mathrm{rad} 7$ and $\mathrm{rad} 16$ also exist, and that they participate in the global repair process. The $\mathrm{rad} 7$ sequence does not reveal any clue to its function [71]. The radl6 gene, however, encodes a protein containing a special type of DNA-binding $\mathrm{Zn}^{2+}$-finger and an extended region with strong homology to a specific subfamily of presumed DNA helicases [72]. Interestingly, a protein specific for transcription-coupled repair, CSB/ERCC6, is also equipped with such domains [24]. A characteristic of other members of this 'helicase' subfamily is that they reside in multiprotein complexes. Genetic evidence in yeast suggests that the complex interacts with chromatin (see [73] and references therein).

In this light, what is the role of the genes mentioned above? One possibility, suggested elsewhere, is that they are involved in uncoupling essential NER components from the transcription machinery to make them available for the global genome repair [69]. Obviously, the BTF2 transcription-repair complex is the most logical partner in this option. The strong ssDNA binding of the XPC complex could release BTF2 from the transcriptioninitiation machinery and make it available for NER. Alternatively, the rad 16 protein(complex?) may be implicated in altering 
chromatin structure required for global genome repair. Obviously, these speculations need to be experimentally investigated.

Steps specific for transcription-coupled repair (Table 3). The only isolated eukaryotic gene that is part of the transcription-coupled repair process is CSB/ERCC6. The not yet cloned gene for the CSA protein is anticipated to participate in this system as well.

CSB/ERCC6 is a member of the closely related subfamily of putative DNA/RNA helicases, described above, to which rad16 also belongs. A yeast homologue of this protein was unknown, but the sequence conservation seen for all other eukaryotic NER factors permitted the large evolutionary gap between man and yeast to be bridged. Like CSB, a yeast disruption mutant (designated rad26) displayed a selective defect in transcriptioncoupled repair [73a]. Interestingly, inactivation of this NER subpathway in yeast does not induce significant UV sensitivity, suggesting that this process is not very important for survival of UV-exposed yeast. It is feasible that CSB/ERCC6 and the other repair members of this 'helicase' subfamily possess a local DNA denaturing activity, thereby inducing displacement of bound proteins (e.g. nucleosomes or a stalled RNA polymerase complex) from the DNA to give repair proteins access to the lesion. The possibility exists that the CSB/ERCC6 protein functions as a general mediator linking transcription to the total of repair pathways. Finally, it should be stressed again that the models proposed here are purely hypothetical and serve to stimulate design of experimental protocols to verify or dismiss them.

\section{INVOLVEMENT OF TRANSRIPTION DEFECTS IN THE COMPLEX NER SYNDROMES}

Clinical heterogeneity and pleiotropy associated with mutations in $B T F 2$

It is clear that the spectrum of diseases linked with mutations in the BTF2 subunits XPB/ERCC3, XPD/ERCC2 and TTDA is remarkably heterogeneous and pleiotropic, and includes seemingly unrelated symptoms, such as photosensitivity, brittle hair and nails, neurodysmyelination, impaired sexual development, ichthyosis and dental caries (see Table 1). The rare XP group B and the more common group D are associated with classical XP (only in group D), atypical combinations of XP and CS, and TTD $[16,74,75]$ (Table 2 ). The clinical variability in TTD is also apparent from the fact that at least 7 disorders arc thought to be identical or closely related to TTD. This includes the Politt, Tay, Sabinas, Netherton, ONMR, Hair-Brain and the Marinesco-Sjögren syndromes [76]. The occurrence of TTD in three distinct NER-deficient complementation groups weighs against a chance association between genetic loci separately involved in NER and in brittle hair. Consistent with the idea that these processes are intimately connected, is the recent identification of point mutations in the XPD/ERCC2 gene in TTD(XP-D) patients [77].

\section{The clinical featues derived from the NER defect}

In striking contrast to the pleiotropy and clinical heterogeneity selectively associated with XP-B and XP-D, the symptoms seen in the most common XP groups A (totally deficient in NER) and $\mathrm{C}$ (defective in the 'genome overall' repair subpathway) are much more uniform (Table 2). They involve only photosensitivity, pigmentation abnormalities, predisposition to skin cancer and, in the case of XP-A, accelerated neurodegeneration (which is not associated with neurodysmyelination), but no CS and TTD manifestations. The gene products affected in these groups are not vital and, therefore, do not appear to be essential for basal transcription. This indicates that these forms of XP present the manifestations of a pure NER defect.

\section{Evidence for the involvement of transcriptional defects in CS and TTD}

The selective association of BTF2 with the peculiar forms of $\mathrm{XP}$ and the dual role of this complex in repair and transcription make it tempting to link the unexpected TTD and CS features with the additional transcription-related function of the NER genes involved. Indeed, it would be highly unlikely that all mutations in the three subunits of this bifunctional complex would affect only the repair function, and leave the inherent transcriptional role intact. This interpretation is supported by the phenotype of a Drosophila ERCC3 mutant. This mutant called haywire displays UV sensitivity, central nervous system abnormalities and impaired sexual development, as found in XP-B [51]. Spermatogenesis in Drosophila is very sensitive to the level of $\beta 2$ tubulin [78]. Mutations in the Drosophila ERCC3 gene seem to affect $\beta$ tubulin expression, causing male sterility [51]. It is, therefore, likely that expression of this gene in Drosophila (and by inference possibly in man) is particularly sensitive to the level of transcription and thereby to subtle mutations in BTF2. This could explain the immature sexual development found in TTD and CS. Expression of the myelin basic protein is known to be critically dependent on transcription. Reduced transcription of this gene has been demonstrated to cause neurological abnormalities in the mouse [79]. Thus, the characteristic neurodysmyelination of CS and TTD $[80,81]$ may also relate to suboptimal transcription of this or other genes involved in myelin sheet formation. Similarly, reduced transcription of genes encoding the class of ultrahigh sulphur proteins of the hairshaft may account for the observed reduced cysteine content in the brittle hair of TTD patients [82]. A comparable explanation is proposed for the poor enamelation of teeth in CS and TTD [13, 83]. The skin abnormalities typical of TTD often involve ichthyosis. Various classes of ichthyoses show abnormalities in the production of filaggrin [84]. Thus, mutations in BTF2 which subtly disturb its transcription function may affect a specific subset of genes whose functioning critically depends on the level or fine-tuning of transcription. Recent studies indicate that the requirement for basal transcription factors may vary from promoter to promoter depending on the sequence around the initiation site, the topological state of the DNA and the local chromatin structure [85-87]. These mechanisms can readily explain the pronounced clinical heterogeneity within families.

As noted previously [17], upon close inspection, many parallels can be found between CS and TTD suggesting that they are manifestations of a broad clinical continuum. This is consistent with the finding that mutations in different subunits of the same (BTF2) complex give rise to a similar set of CS and TTD features. Thus, defects in CSA and CSB/ERCC6 [24] as well as $X P G / E R C C 5$ [12] may also affect basal transcription, as they give rise to a comparable phenotype.

\section{Combined repair/transcription and sole transcription syndromes}

A tentative model proposed for the aetiology of the defects in the conglomerate of CS, TTD and related disorders is shown in Table 4. In this model, mutations in BTF2 subunits inactivating only the NER function result in a XP phenotype as observed in the classical XP patients of XP group D. If, in addition the transcription function is subtly affected, the combination of XP 
Table 4. Dual function of BTF2/TFIIH in transcription and $N E R$-possible consequences for human disorders

\begin{tabular}{lll}
\hline Function & Syndrome & Category disorder \\
\hline $\begin{array}{l}\text { NER inactivated } \\
\text { Transcription normal }\end{array}$ & XP* & Repair \\
$\begin{array}{l}\text { NER inactivated } \\
\text { Transcription partially } \\
\text { inactivated }\end{array}$ & PIBIDS (XP/CS) $\dagger$ & Transcription/Repair \\
$\begin{array}{l}\text { NER normal } \\
\text { Transcription partially }\end{array}$ & TTD $\dagger$ & \\
inactivated & & Transcription \\
$\begin{array}{l}\text { NER normal or } \\
\text { inactivated } \\
\text { Transcription } \\
\text { inactivated } \\
end{array}$ & (Lethal) & - \\
\hline
\end{tabular}

Model for the involvement of defects in transcription and nucleotide excision repair in the human disorders xeroderma pigmentosum, Cockayne syndrome, trichothiodystrophy and a number of related diseases. See text for further explanation.

* NER features: photosensitivity, increased frequency of skin cancer, neurodegeneration. $\nmid$ Transcription features: brittle hair (cysteine-rich matrix protein), neurodysmyelination (myelin proteins), decreased fertility (testis-spec. $\beta$ - 2 tubulin?), poor growth, ich thyosis (cornified envelope protein?). ‡ Transcription function subtly affected so mutants still viable. $\$$ Transcription function completely inactivated.

and CS or TTD is found. Theoretically, mutations causing a (still viable) transcription problem without NER impairment may be expected as well. Indeed, a significant proportion of TTD and clinically characteristic CS patients are not noticeably photosensitive and have normal NER $[88,89]$. These findings extend the implications to non-repair-defective disorders. Therefore, and in view of the pronounced heterogeneity inherent to the model, it is possible that the Siögren-Larsson (270200), RUD (308200), ICE (146720), OTD (257960), IFAP (308205), CAM(F)AK (214550), Rothmund-Thompson (268400) and KID (242150) syndromes (for references: $[76,90]$ ) also fall within this category. Interestingly, some of these diseases show occurrence of skin cancer.

In conclusion, these findings provide evidence for the presence of a wide class of disorders that collectively can be called "transcription syndromes". A prediction from our model is that these patients carry mutations in transcription factors, that do not affect the NER process. This explanation introduces a novel concept into human genetics. It can be envisaged that similar phenomena are associated with subtle defects in translation, implying the potential existence of "translation syndromes".

\section{CONCLUDING REMARKS}

After a long period of stagnation, the field of NER has dramatically gained momentum in recent years and is poised for further breakthroughs. The basis for this progress has been laid by the application of recombinant DNA technology, permitting cloning and characterisation of a large set of NER genes, the development of a bona fide in vitro assay and the discovery of unexpected links with other processes. These findings can now be tentatively integrated into a possible model for the complex NER reaction. Ahead of us lies the task of unravelling this intricate mechanism by filling in the gaps in our perception and fitting the pieces of the puzzle together. However, it is not difficult to foresee that, within the near future, the core of the NER reaction will be reconstituted in vitro using purified components. Detailed understanding of the molecular mechanism of NER is crucial for gaining insight into the specificity, efficiency and fidelity of this important pathway for genome stability and prevention of cancer. Furthermore, the puzzling features associated with a specific subset of NER syndromes have found an unexpected but plausible molecular explanation by the intimate connection with transcription. The application of powerful methodologies to manipulate the mammalian genome will allow the generation of a series of mouse models for all three NER syndromes. The development of the first NERcompromised mouse models has already been welcomed [91] (Tanaka, personal communication; Weeda et al., unpublished results). This will be of great importance for clinical studies, and to assess the biological relevance of the NER system and for cancer research in general.

1. Friedberg EC. DNA Repair. San Francisco, Freeman WH and Company, 1985

2. Sancar A, Sancar GB. DNA repair enzymes. Ann Rev Biochem $1988,57,29-67$.

3. Van Houten B. Nucleotide excision repair in Escherichia coli. Microbiol Rev 1990, 54, 18-51.

4. Grossman L, Thiagalingam S. Nucleotide excision repair, a tracking mechanism in search of damage. $\mathcal{J}$ Biol Chem 1993, 268, 16871-16874.

5. Hoeijmakers JHJ. Nucleotide excision repair I: from $E$. coli to yeast. TIG 1993, 9, 173-177.

6. Hanawalt PC, Mellon I. Stranded in an active gene. Curr Biol 1993, 3, 67-69.

7. Bohr VA. Gene specific DNA repair. Carcinogenesis 1991, 12, 1983-1992.

8. Vermeulen W, Stefanini M, Giliani S, Hoeijmakers JHJ, Bootsma $D$. Xeroderma pigmentosum complementation group $\mathrm{H}$ falls into complementation group C. Mutat Res 1991, 255, 201-208.

9. Cleaver JE, Kraemer KH. Xeroderma pigmentosum. In Scriver CR, Beaudet AL, Sly WS, Valle D. eds. The Metabolic Basis of Inherited Disease, seventh edition. New York, McGraw-Hill Book Co., 1994.

10. Tanaka K, Kawai K, Kumahara Y, Ikenaga M, Okada Y. Genetic complementation groups in Cockayne syndromc. Somatic Cell Genet $1981,7,445-455$.

11. Lehmann AR. Three complementation groups in Cockayne syndrome. Mutat Res 1982, 106, 347-356.

12. Vermeulen W, Jaeken J, Jaspers NGJ, Bootsma D, Hoeijmakers $\mathrm{JHJ}$. Xeroderma pigmentosum complementation group $\mathrm{G}$ associated with Cockayne's syndrome. Am f Human Genet 1993, 53, 185-192.

13. Nance MA, Berry SA. Cockayne syndrome: review of 140 cases $A m$ 7 Med Genet 1992, 42, 68-84.

14. Stefanini $M$, Giliani S, Nardo T, et al. DNA repair investigations in nine Italian patients affected by trichothiodystrophy. Mutat Res $1992,273,119-125$

15. Stefanini $M$, Vermeulen $W, W e e d a ~ G$, et al. A new nucleotideexcision-repair gene associated with the disorder trichothiodystrophy. Am f Human Genet 1993, 53, 1-5.

16. Vermeulen W, van Vuuren AJ, Chipoulet $M$, et al. Three unusual repair deficiencies associated with transcription factor BTF2(TFIIH). Evidence for the existance of a transcription syndrome. Cold Spring Harbor Symp Quant Biol 1994, 57, in press.

17. Bootsma D, Hoeijmakers JHJ. Engagement with transcription. Nature 1993, 363, 114-115.

18. Numata $M$, Hata $H$, Shiomi $T$, et al. Identification of the cellular defect in UVS1, a UV-sensitive Chinese hamster ovary mutant cell line. Cancer Res 1993, 53, 495-499.

19. Busch D, Greiner C, Rosenfeld KL, et al. Complementation group assignments of moderately UV-sensitive $\mathrm{CHO}$ mutants isolated by large-scale screening. Mutagenesis 1994, 9, 301-306.

20. Flejter WL, McDaniel LD, Johns D, Friedberg EC, Schultz RA. Correction of xeroderma pigmentosum complementation group D mutant cell phenotypes by chromosome and gene transfer: 
involvement of the human ERCC2 DNA repair gene. Proc Natl Acad Sci USA 1992, 89, 261-265.

21. Weeda G, Van Ham RCA, Vermeulen W, Bootsma D, Van der Eb AJ, Hoeijmakers JHJ. A presumed DNA helicase encoded by $E R C C-3$ is involved in the human repair disorders xeroderma pigmentosum and Cockayne's syndrome. Cell 1990, 62, 777-791.

22. Scherly D, Nouspikel T, Corlet J, Ucla C, Bairoch A, Clarkson SG. Complementation of the DNA repair defect in xeroderma pigmentosum group $\mathrm{G}$ cells by a human cDNA related to yeast RAD2. Nature 1993, 363, 182-185.

23. O'Donovan A, Wood RD. Identical defects in DNA repair in xcrodcrma pigmentosum group $\mathrm{G}$ and rodent ERCC group 5. Nature 1993, 363, 185-188.

24. Troelstra C, van Gool A, de Wit J, Vermeulen W, Bootsma D, Hoeijmakers JHJ. ERCC6, a member of a subfamily of putative helicases, is involved in Cockayne's syndrome and preferential repair of active genes. Cell 1992, 71, 939-953.

25. Van Duin M, Vredeveldt G, Mayne LV, et al. The cloned human DNA excision repair gene $E R C C-1$ fails to correct xeroderma pigmentosum complementation groups A through I. Mutat Res $1989,217,83-92$.

26. van Vuuren AJ, Appeldoorn E, Odijk H, et al. Evidence for a repair enzyme complex involving ERCC1 and complementing activities of ERCC4, ERCC11 and xeroderma pigmentosum group F. EMBOf 1993, 12, 3693-3701.

27. Biggerstaff $M$, Szymkowski DE, Wood RD. Co-correction of ERCCl, ERCC4 and xeroderma pigmentosum group F DNA repair defects in vitro. EMBOF 1993, 12, 3685-3692.

27a. Hoeijmakers JH. Nucleotide excision repair II: from yeast to mammals. Trends Genet 1993, 9, 173-177.

28. Friedbery EC. Deoxyribunucleic acid repair in the yeast Saccharomyces cerevisiae. Microbiol Rev 1988, 52, 70-102.

29. Prakash S, Sung P, Prakash L. DNA repair genes and proteins of Saccharomyces cerevisiae. Ann Rev Genet 1993, 27, 33-70.

30. Venema J, van Hoffen A, Karcagi V, Natarajan AT, van Zeeland AA, Mullenders LHF. Xeroderma pigmentosum complementation group $\mathrm{C}$ cells remove pyrimidine dimers selectively from the transcribed strand of active genes. Mol Cell Biol 1991, 11, 4128-4134.

31. Venema J, Mullenders LHF, Natarajan AT, Van Zccland $\Lambda \Lambda$, Mayne LV. The genetic defect in Cockayne syndrome is associated with a defect in repair of UV-induced DNA damage in transcriptionally active DNA. Proc Natl Acad Sci USA 1990, 87, 4707-4711.

32. van Hoffen A, Natarajan AT, Mayne LV, van Zeeland A, Mullenders LHF, Venema J. Deficient repair of the transcribed strand of active genes in Cockayne's syndrome cells. Nucleic Acids Res 1993, 21, 5890-5895.

33. Tanaka K, Miura N, Satokata I, et al. Analysis of a human DNA excision repair gene involved in group A xeroderma pigmentosum and containing a zinc-finger domain. Nature 1990, 348, 73-76.

34. Hoeijmakers JHJ, Eker APM, Wood RD, Robins P. Use of in vivo and in vitro assays for the characterization of mammalian excision repair and isolation of repair proteins. Mutat Res 1990, 236, 223-238.

35. Robins $\mathrm{P}$, Jones CJ, Biggerstaff $M$, Lindahl T, Wood RD. Complementation of DNA repair in xeroderma pigmentosum group A cell extracts by a protein with affinity for damaged DNA. EMBOF 1991, 10, 3913-3921.

36. Eker APM, Vermeulen W, Miura N, et al. Xeroderma pigmentosum group A correcting protein from calf thymus. Mutat Res 1992, 274, 211-224.

37. Miyamoto I, Miura N, Niwa H, Miyazaki J, Tanaka K. Mutational analysis of the structure and function of the xeroderma pigmentosum group A complementing protein. $\mathrm{F} \mathrm{Biol} \mathrm{Chem} \mathrm{1992,} \mathrm{267,}$ 12182-12187.

38. Jones CJ, Wood RD. Preferential binding of the xeroderma pigmentosum group A complementing protein to damaged DNA. Biochemistry 1993, 32, 12096-12104.

39. Shivji MKK, Kenny MK, Wood RD. Proliferating cell nuclear antigen is required for DNA excision repair. Cell 1992, 69, 367-374.

40. Hwang BJ, Chu G. Purification and characterization of a human protein that binds to damaged DNA. Biochemistry 1993, 32, $1657-1666$

41. van Assendelft GB, Rigney EM, Hickson ID. Purification of a HeLa cell nuclear protein that binds selectively to DNA irradiated with Ultra-Violet light. Nucleic Acids Res 1993, 21, 3399-3404.

42. Keeney S, Chang GJ, Linn S. Characterization of a human DNA damage binding protein implicated in xeroderma pigmentosum $\mathrm{E}$. 7 Biol Chem 1993, 268, 21293-21300.

43. Takao M, Abramic M, Moos M, et al. A $127 \mathrm{kDa}$ component of a UV-damaged DNA-binding complex which is defective in some xeroderma pigmentosum group $\mathbf{E}$ patients is homologous to a slime mold protein. Nucleic Acids Res 1993, 21, 4111-4118.

44. Keeney S, Eker APM, Brody T, et al. Correction of the DNA repair defect in xeroderma pigmentosum group $\mathrm{E}$ of a DNA damagebinding protein. Proc Natl Acad Sci IISA 1994, 91, 4053-4056.

45. Reardon JT, Nichols AF, Keeney S, et al. Comparative analysis of binding of human damage DNA-binding protein (XPE) and Escherichia coli damage recognition protein (UvrA) to the major ultraviolet photoproducts - T[CS]TT[Ts]TT[6-4]T and T[Dewar]T. F Biol Chem 1993, 268, 21301-21308.

46. Li L, Elledge SJ, Peterson CA, Bales ES, Legerski RJ. Specific association between the human DNA repair proteins XPA and ERCC1. Proc Natl Acad Sci USA 1994, 91, 5012-5016.

47. Park C-H, Sancar A. Formation of a ternary complex by human $\mathrm{XPA}, \mathrm{ERCCl}$ and ERCC4(XPF) excision repair proteins. Proc Natl Acad Sci USA 1994, 91, 5017-5021.

48. Coverley D, Kenny MK, Lane DP, Wood RD. A role for the human single-stranded DNA binding protein HSSB/RPA in an early stage of nucleotide excision repair. Nucleic Acids Res 1992, 20, 3873-3880.

49. Naumovski L, Friedberg EC. A DNA repair gene required for the incision of damaged DNA is essential for viability in Saccharomyces cerevisiae. Proc Nall Acad Sci USA 1983, 80, 4818-4821.

50. Park E, Guzder S, Koken MHM, et al. RAD25, a yeast homolog of human xeroderma pigmentosum group B DNA repair gene is essential for viability. Proc Natl Acad Sci USA 1992, 89, $11416-11420$

51. Mounkes LC, Jones RS, Liang B-C, Gelbart W, Fuller MT. A Drosophila model for xeroderma pigmentosum and Cockayne's syndrome: haywire encodes the fly homolog of $E R C C 3$, a human excision repair gene. Cell 1992, 71, 925-937.

52. Schaeffer I., Roy R, Humbert S, et al. DNA repair helicase: a component of BTF2 (TFIIH) basic transcription factor. Science $1993,260,58-63$.

53. Conaway RC, Conaway JW. General initiation factors for RNA polymerase II. Ann Rev Biochem 1993, 62, 161-190.

54. Gerard M, Fischer L, Moncollin V, Chipoulet J-M, Chambon P, Egly J-M. Purification and interaction properties of the human RNA polymerase B(II) general transcription factor BTF2. 7 Biol Chem 1991, 266, 20940-20945.

55. Conaway RC, Conaway JW. An RNA polymerase II transcription factor has an associated DNA-dependent ATPase (dATPase) activity strongly stimulated by the TATA region of promoters. Proc Natl Acad Sci USA 1989, 86, 7356-7360.

56. Schaeffer L, Moncollin V, Roy R, et al. The ERCC2/DNA repair protein is associated with the class II BTF2/TFIIH transcription factor. $E M B O F$ I 1994, 13, 2388-2392.

57. Drapkin R, Reardon JT, Ansari A, et al. Dual role of the TFIIH in DNA excision repair and in transcription by RNA polymerase II. Nature 1994, 368, 769-772.

58. van Vuuren AJ, Vermeulen $\mathrm{W}, \mathrm{Ma} \mathrm{L}$, et al. Correction of xeroderma pigmentosum repair defect by basal transcription factor BTF2(TFIIH). EMBO 7 1994, 13, 1645-1653.

59. Humbert S, van Vuuren AJ, Lutz Y, Hoeijmakers JHJ, Egly J-M, Moncollin V. Characterization of p44/SSL1 and p34 subunits of the BTF2/TFIIH transcription/repair factor. EMBO 7 1994, 13, 2393-2398.

60. Yoon H, Miller SP, Pabich EK, Donahue TF. SSL1, a suppressor of a HIS4 5'-UTR stem-loop mutation, is essential for translation initiation and effects UV resistance in yeast. Genet Devel 1992, 6, 2463-2477.

61. Goodrich JA, Tjian R. Transcription factors IIE and IIH and ATP hydrolysis direct promoter clearance by RNA polymerase II. Cell $1994,77,145-156$

62. Huang JC, Svoboda DL, Reardon JT, Sancar A. Human nucleotide excision nuclease removes thymine dimers from DNA by incising the 22nd phosphodiester bond $5^{\prime}$ and the 6th phosphodiester bond $3^{\prime}$ to the photodimer. Proc Natl Acad Sci USA 1992, 89, 3664-3668.

63. Sung P, Reynolds P, Prakash L, Prakash S. Purification and characterization of the Saccharomyces cerevisiae RAD1/RAD10 endonuclease. F Biol Chem 1993, 268, 26391-26399.

64. Habrakien Y, Sung P, Prakash L, Prakash S. Yeast excision repair 
gene $R A D 2$ encodes a single-stranded DNA endonuclease. Nature $1993,366,365-368$.

65. Harrington JJ, Lieber NIR. Functional domains within FEN-1 and RAD2 define a family of structure-specific endonucleases: implications for nucleotide excision repair. Genet Devel 1994, 8, 1344-1355.

66. Nichols AF, Sancar A. Purification of PCNA as a nucleotide excision repair protein. Nucleic Acids Res 1992, 20, 2441-2446.

67. Verhage $\mathrm{R}$, Zeeman $\mathrm{A}$, de: Groot $\mathrm{N}$, et al. The RAD7 and $R A D 16$ genes are essential for repair of non-transcribed DNA in Saccharomyces cerevisiae. Mol Cell Biol 1994, 14, 6135-6142.

68. Legerski R, Peterson C. Expression cloning of a human DNA repair gene involved in xeroderna pigmentosum group C. Nature 1992, $359,70-73$.

69. Masutani C, Sugasawa K., Yanagisawa J, et al. Purification and cloning of a nucleotide excision repair complex involving the xeroderma pigmentosum group $\mathrm{C}$ protein and a human homolog of yeast RAD23. EMBO f 1994, 13, 1831-1843.

70. van der Spek PJ, Smit EME, Beverloo HB, et al. Chromosomal localization of three repair genes: xeroderma pigmentosum group $\mathrm{C}$ and two human homologs of yeast RAD23. Genomics 1994, 23, 651-658.

71. Perozzi G, Prakash S. RAD7 of Saccharomyces cerevisiae: transcripts, nucleotide sequence analysis and functional relationship between the RAD7 and RAD23 gene products. Mol Cell Biol 1986, 6, 1497-1507.

72. Bang DD, Verhage R, Goosen N, Brouwer J, Putte Pvd. Molecular cloning of $R A D 16$, a gene involved in differential repair in Saccharomyces cerevisiae. Nucleic Acids Res 1992, 20, 3925-3931.

73. Wolffe AP. Switched-on chromatin. Curr Biol 1994, 4, 525-528.

73a. Gool AJ van, Verhage R, Swagemakers SMA, et al. RAD26, the functional $S$. cerevisiae homolog of the Cockayne syndrome B gene ERCC6. Embo 7 1994, in press.

74. Johnson RT, Squires S. The XPD complementation group. Insights into xeroderma pigmentosum, Cockayne's syndrome and trichothiodystrophy. Mutat Res 19912, 273, 97-118.

75. Vermeulen W, Scott RJ, Potger S, et al. Clinical heterogeneity within xeroderma pigmentosum associated with mutations in the DNA repair and transcription gene ERCC3. Am f Human Genet 1994, 54, 191-200.

76. McKusick VA. Mendelian Inheritance in Man. Catalogs of Autosomal Dominant, Autosomal Receisive and X-linked Phenotypes. Baltimore, The John Hopkins University Press, 1992.

77. Broughton BC, Steingrimsdottir H, Weber CA, Lehmann AR. Mutations in the xeroderrna pigmentosum group D DNA repair/ transcription gene in patients with trichothiodystrophy. Nature Genet 1994, 7, 189-194.

78. Kemphues KJ, Kaufmar TC, Raff RA, Raff EC. The testis- specific beta-tubulin subunit in Drosophila melanogaster has multiple functions in spermatogenesis. Cell 1982, 31, 655-670.

79. Readhead C, Popko B, Takahashi N, et al. Expression of a myelin basic protein gene in transgenic shiverer mice: correction of the dysmyelinating phenotype. Cell 1987, 48, 703-712.

80. Peserico A, Battistella PA, Bertoli P. MRI of a very rare hereditary ectodermal dysplasia: PIBI(D)S. Neuroradiology 1992, 34, 316-317.

81. Sasaki K, Tachi N, Shinoda M, Satoh N, Minami R, Ohnishi A. Demyelinating peripheral neuropathy in Cockayne syndrome: a histopathologic and morphometric study. Brain Dev 1992, 14, 114-117.

82. Itin PH, Pittelkow MR. Trichothiodystrophy: review of sulfurdeficient brittle hair syndromes and association with the ectodermal dysplasias. 7 Am Acad Dermatol 1990, 22, 705-717.

83. McCuaig C, Marcoux D, Rasmussen JE, Werner MM, Genter NE. Trichothiodystrophy associated with photosensitivity, gonadal failure, and striking osteosclerosis. 7 Am Acad Dermatol 1993, 28, 820-826.

84. Fleckman P, Dale BA. Pharmacology of the Skin. Bernard BA, Shroot B. eds. Basel, Karger Medical and Scientific Publishers, 1993 , vol. 15.

85. Parvin JD, Sharp PA. DNA topology and a minimum set of basal factors for transcription by RNA polymerase II. Cell 1993, 73, 533-540.

86. Stanway CA. Simplicity amidst complexity in transcriptional initiation. Bioessays 1993, 15, 559-560.

87. Timmers HTM. Transcription initiation by RNA polymerase II does not require hydrolysis of the beta-gamma phosphoanhydride bond of ATP. EMBOF 1994, 13, 391-399.

88. Lehmann AR. Cockayne's syndrome and trichothiodystrophy: defective repair without cancer. Cancer Rev 1987, 7, 82-103.

89. Lehmann AR, Thompson AF, Harcourt SA, Stefanini M, Norris PG. Cockayne's syndrome: correlation of clinical features with cellular sensitivity of RNA synthesis to UV irradiation. $f$ Med Genet $1993,30,679-682$.

90. Baden H. Keratinizing disorders. In Alpen JC, eds. Genetic Disorders of the Skin. Mosby Yearbook Inc., 1991, pp 170-194.

91. McWhir J, Selfridge J, Harrison DJ, Squires S, Melton DW. Mice with DNA repair gene (ERCCI) deficiency have elevated levels of p53, liver nuclear abnormalities and die before weaning. Nature Genet 1993 4, 217-223.

Acknowledgements - I am indebted to all members of the DNA repair group of the Medical Genetics Centre for valuable and stimulating discussions. Research in our group is supported by the Netherlands Organization for the Advancement of Pure Science, through the Foundation of Medical Scientific Research (grant 900-501-91, 93, 113), the Dutch Cancer Society, and Euratom.

\section{Genetic Polymorphisms in Xenobiotic Metabolism}

\section{C.A.D. Smith, G. Smith and C.R. Wolf}

\section{INTRODUCTION}

ThE MULTI-STEP or multi-hit theory of carcinogenesis states that tumour formation is due to the consequences of multiple mutagenic events. An obvious conclusion to be drawn from this is that predisposition to cancer, due to inherited genetic factors, must have an enormous impact on the development of disease.

Correspondence to C.A.D. Sinith at the Department of Pathology, University of Edinburgh, Edinburgh EH8 9AG, U.K

G. Smith and C.R. Wolf aris at the Biomedical Research Centre, Ninewells Hospital and Medical School, Dundee DDI 9SY, U.K.
Indeed, intense study of familial cancer, childhood neoplasms, onco-viruses and animal models have now identified a large number of candidate genes that directly influence the aetiology and pathogenesis of many cancers. Although there is no doubt that these cancer genes have an important role in tumorigenesis, the vast majority of cancers do not appear to have any strong familial link. It is now widely accepted that the initiation and development of tumours are determined by a delicate balance of environmental and host factors. 